\title{
Combined TOA/DOA for Location Estimation in MIMO System
}

\author{
A. A. M. Isa ${ }^{*}$, M. H. Othmana, N. Z. Harona, M. S. M. Isaa, M. S. I. M. Zina, Z. Zakaria ${ }^{a}$, A. A. A. Zainib \\ ${ }^{a}$ Centre for Telecommunication Research and Innovation (CeTRI), Faculty of Electronic and Computer Engineering, Universiti Teknikal Malaysia Melaka, \\ Malaysia \\ ${ }^{b}$ Department of Mechanical Engineering, Politeknik Merlimau, Melaka, Malaysia \\ *Corresponding author: azmiawang@utem.edu.my
}

\section{Article history}

Received :1 January 2014

Received in revised form:

15 February 2014

Accepted :18 March 2014

\section{Graphical abstract}

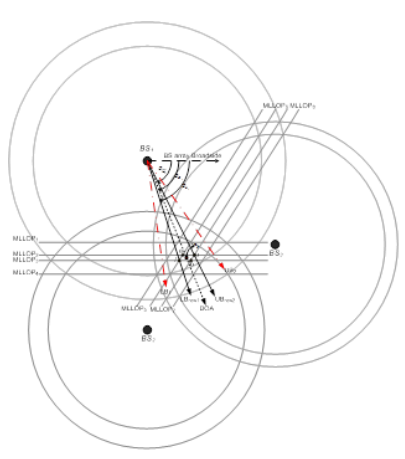

\section{Abstract}

This paper proposes on the development of a combined location and positioning (L\&P) system by combining range measurements and angle measurements for a multiple input multiple output (MIMO) system. The proposed combined technique called as hybrid multiple linear lines of position (HMLLOP) algorithm extends the fundamental idea of using the multiple linear lines of position (MLLOP) scheme when extra information in the form of direction of arrival (DOA) measurements at a minimum of three base stations (BSs) is available. The technique explores the use of multiple lines of position (LOP) instead of circular LOP and determines new lower and upper bounds of DOA according to the measurements obtained from an MLLOP scheme which aims to minimize the DOA error caused by non-line of sight (NLOS) propagation. Simulation results have been provided that show that the proposed combined schemes outperform the range-only algorithm in terms of estimated location accuracy. It has been shown that time of arrival (TOA)-based location can be employed as a baseline location estimation technique, with additional DOA measurements being used to further improve accuracy.

Keywords: Direction of arrival; location and positioning; MIMO

C) 2014 Penerbit UTM Press. All rights reserved.

\subsection{INTRODUCTION}

Hybrid L\&P algorithms which employ the combinations of available range, and angle of arrival (AOA) measurements to estimate the mobile station (MS) position in a wireless communication system. The technique is very helpful in certain application scenarios, especially when the number of BSs is limited.

MS position can be determined using various parameters such as signal strength (SS), AOA, TOA, time difference of arrival (TDOA), combined methods, etc. [1-3]. Among them, combined location systems are more popular because of their potential for estimating location with high accuracy. It is noted that a major problem that affects the accuracy of mobile location estimates is NLOS propagation, where the absence of a direct LOS path between BS and MS results in biased measurements and produces inaccurate positioning in the estimation of MS location, no matter which method is utilized. NLOS propagation results in time and angle measurements that have large errors due to single or multiple reflections and diffraction of the signal between the MS and BS. For direction finding location systems, the angle from which the signal arrives at the MS does not represent the true direction of the BS. This can lead to a severe degradation in positioning accuracy if standard LOS-based location estimation algorithms are employed.

As described in [4] WiMAX technology supports several multiple-antenna technologies, such as smart antenna systems, beamforming and MIMO. Recently, combination of both beamforming and MIMO technologies have been utilized for mobile location scenario [5]. MIMO may be utilized when available at BSs and MS to improve location estimation accuracy $[6,7]$. MIMO can also be combined with beamforming to offer optimal estimation accuracy results [8]. By exploiting the multipath characteristics of MIMO and beamforming, it is possible to determine the position of the MS by considering the capability of MIMO to mitigate NLOS conditions. In a wireless system, parameters such as the TOA and DOA of multipath signals can be estimated by using advanced array signal processing techniques such as in [9].

In this paper, a combined L\&P technique is proposed which determine the position of the MS based on a combination of a MLLOP range-based algorithm and DOA-based beamforming. As proposed in [10], the use of an MLLOP scheme increases the efficiency of the proposed scheme. The proposed combined technique augments the fundamental idea of using an MLLOP scheme when extra information in the form of DOA measurements at a minimum of three BSs is available. The technique explores the 
use of multiple LOP instead of circular LOP and determines new lower and upper bounds for DOA according to the measurements obtained from an MLLOP scheme which aims at minimizing the DOA error caused by NLOS propagation.

\subsection{REVIEW OF COMBINED TOA-DOA USING LLS/NLLS (HTD)}

The following is a two-step combined TOA/DOA-based beamforming procedure proposed by Sayed et al. [11] whereby a TOA procedure uses LLS and NLLS algorithms, and an AOA procedure applies an LLS algorithm. We assume that $N$ BSs estimate the TOA and AOA of the MS. From the TOA equation, the LLS estimate of the MS position using TOA measurements is given by [12]:

$\boldsymbol{\theta}_{T O A}=\left(\boldsymbol{A}_{T O A}^{T} \boldsymbol{A}_{T O A}\right)^{-1} \boldsymbol{A}_{T O A}^{T} \boldsymbol{b}_{T O A}+\boldsymbol{\beta}_{T O A}$

where:

$$
\begin{aligned}
& \boldsymbol{\theta}_{T O A}=\left[\begin{array}{l}
x_{u} \\
y_{u}
\end{array}\right] \\
& \boldsymbol{\beta}_{T O A}=\left[\begin{array}{l}
x_{1} \\
y_{1}
\end{array}\right] \\
& \boldsymbol{A}_{T O A}=\left[\begin{array}{cc}
x_{2}-x_{1} & y_{2}-y_{1} \\
x_{3}-x_{1} & y_{3}-y_{1} \\
\vdots & \vdots \\
x_{n}-x_{1} & y_{n}-y_{1}
\end{array}\right] \\
& \boldsymbol{b}_{T O A}=\left[\begin{array}{l}
b_{21} \\
b_{31} \\
b_{n 1}
\end{array}\right] \\
& b_{i j_{T O A}}=0.5\left[r_{j}^{2}-r_{i}^{2}+d_{i j}^{2}\right]
\end{aligned}
$$

Meanwhile, the NLLS estimate of the MS position using TOA measurement is then given by $[12,13]$ :

$$
\boldsymbol{\theta}_{\{k\} T O A}=\boldsymbol{\theta}_{\{k\} T O A}-\left(\boldsymbol{J}_{\{k\} T O A}^{T} \boldsymbol{J}_{\{k\} T O A}\right)^{-1} \boldsymbol{J}_{\{k\} T O A}^{T} \boldsymbol{f}_{\{k\} T O A}
$$

where:

$$
\begin{gathered}
\boldsymbol{\theta}_{\text {TOA }}=\left[\begin{array}{l}
x_{u} \\
y_{u}
\end{array}\right] \\
\boldsymbol{J}^{T} \boldsymbol{J}_{T O A}=\left[\begin{array}{cc}
\sum_{i=1}^{n} \frac{\left(x-x_{i}\right)^{2}}{\left(f_{i}+r_{i}\right)^{2}} & \sum_{i=1}^{n} \frac{\left(x-x_{i}\right)\left(y-y_{i}\right)}{\left(f_{i}+r_{i}\right)^{2}} \\
\sum_{i=1}^{n} \frac{\left(x-x_{i}\right)\left(y-y_{i}\right)}{\left(f_{i}+r_{i}\right)^{2}} & \sum_{i=1}^{n} \frac{\left(y-y_{i}\right)^{2}}{\left(f_{i}+r_{i}\right)^{2}}
\end{array}\right] \\
\boldsymbol{J}^{T} \boldsymbol{f}_{T O A}=\left[\begin{array}{l}
\sum_{i=1}^{n} \frac{\left(x-x_{i}\right) f_{i}}{\left(f_{i}+r_{i}\right)} \\
\sum_{i=1}^{n} \frac{\left(y-y_{i}\right) f_{i}}{\left(f_{i}+r_{i}\right)}
\end{array}\right]
\end{gathered}
$$

Similarly, the LLS estimate of the MS position using only DOA measurements is given by [12]:

$$
\boldsymbol{\theta}_{A O A}=\left(\boldsymbol{A}_{A O A}^{T} \boldsymbol{A}_{A O A}\right)^{-1} \boldsymbol{A}_{A O A}^{T} \boldsymbol{b}_{A O A}
$$

where:

$$
\begin{gathered}
\boldsymbol{\theta}_{A O A}=\left[\begin{array}{l}
x_{u} \\
y_{u}
\end{array}\right] \\
\boldsymbol{b}_{A O A}=\left[\begin{array}{c}
x_{1}+R_{1} \cos \beta_{1} \\
y_{1}+R_{1} \sin \beta_{1} \\
\hline x_{2}+R_{2} \cos \beta_{2} \\
y_{2}+R_{2} \sin \beta_{2} \\
\hline \vdots \\
\hline x_{i}+R_{i} \cos \beta_{i} \\
y_{i}+R_{i} \sin \beta_{i}
\end{array}\right]
\end{gathered}
$$

$$
\boldsymbol{A}_{A O A}=\left[\begin{array}{cc}
1 & 0 \\
0 & 1 \\
\hline 1 & 0 \\
0 & 1 \\
\hline \vdots & \vdots \\
\hline 1 & 0 \\
0 & 1
\end{array}\right]
$$

The final location estimate can be taken as being a combination of the two estimates (TOA and DOA) as following:

$$
\boldsymbol{\theta}_{\text {Hybrid }}=\gamma \boldsymbol{\theta}_{T O A}+(1-\gamma) \boldsymbol{\theta}_{A O A}
$$

where a parameter $\gamma$ is selected depending on the corresponding accuracy of the TOA and DOA measurements. The value of $\gamma$ is bounded by $0 \leq \gamma \leq 1$.

\subsection{PROPOSED COMBINED MULTIPLE LINEAR LINES} OF POSITION

The above combined method, assumes that TOA generates circular lines of position (CLOP) and combines these with additional information of DOA to determine MS. The system accuracy can be further improved by using MLLOP algorithms that generate multiple LOPs instead of CLOP for the MS by differencing pairs of squared range estimates and proceeding to solve the MS position using a geometric approach or least squares method [10].

In this section a combined L\&P technique is proposed by extending the basic idea of utilizing the MLLOP approach when additional information in the form of DOA-based beamforming measurements at available BSs. This combined technique is called a HMLLOP algorithm. The proposed HMLLOP employs DOA measurements at $N$ MIMO BSs, including the home BS, and attempts to emulate the methodology adopted for MLLOP using the TOA measurements [10] to calculate the MS position. We consider the case where the DOA measurements are available at $N$ MIMO BSs in a macro-cellular environment. In this combined technique, besides employing MLLOP in TOA-based location estimation, errors in DOA can also be minimized by the estimation of new lower bound (LB) and upper bound (UB) values which are 
obtained from the MLLOP scheme. Figure 1 shows the geometry of the proposed algorithm implemented in a MIMO2x1 antenna mode configuration at three BSs including the serving BS. For simplicity, we assume that all available BSs have the same MIMO antenna mode configurations.

Let us assume $N$ BSs with MIMO capability, positioned at $\left\{\boldsymbol{x}_{i}=\left[x_{i}, y_{i}\right]^{T} ; i=1,2, \ldots, N\right\}$, which acquire TOA and DOA measurements from a communication channel with an MS, and that each TOA measurement between each MIMO antenna at $B S_{i}$ and MS is denoted by $\delta_{i, n}$ for $n=1,2, \ldots, N_{t} \times N_{r}$, where $N_{t}$ is the number of transmitter antennae; $N_{r}$ is the number of receiver antennae, and each DOA-based beamforming measurement between MIMO $B S_{i}$ and the MS is denoted by $\theta_{D_{i}}$. Recall that for a MIMO2x1 antenna system employing an MLLOP technique producing a total of 16 intersections points of estimation for MS by using TOA measurements, the possible estimates of the MS at the intersection points can be calculated as follows:

$\hat{\boldsymbol{x}}=\left(\boldsymbol{H}^{T} \boldsymbol{H}\right)^{-1} \boldsymbol{H}^{T} \boldsymbol{B}$

Where:

$\boldsymbol{H}=\left[\begin{array}{cc}C_{1} & 0 \\ 0 & C_{1} \\ \overline{C_{2}} & 0 \\ 0 & C_{2} \\ \hline \vdots & \vdots \\ C_{N-1} & 0 \\ 0 & C_{N-1}\end{array}\right], \quad \boldsymbol{x}=\left[\begin{array}{c}x_{e_{n}} \\ y_{e_{n}}\end{array}\right]$,

$\boldsymbol{B}=\left[\begin{array}{c}B_{1}\left(y_{1}-y_{2}\right)-A_{1}\left(y_{1}-y_{3}\right) \\ A_{1}\left(x_{1}-x_{3}\right)-B_{1}\left(x_{1}-x_{2}\right) \\ \overline{B_{2}\left(y_{1}-y_{3}\right)-A_{2}\left(y_{1}-y_{4}\right)} \\ A_{2}\left(x_{1}-x_{4}\right)-B_{2}\left(x_{1}-x_{3}\right) \\ \vdots \\ B_{N-1}\left(y_{1}-y_{N-1}\right)-A_{N-1}\left(y_{1}-y_{N}\right) \\ A_{N-1}\left(x_{1}-x_{N)}\right)-B_{N-1}\left(x_{1}-x_{N-1}\right)\end{array}\right]$

and :

$$
\begin{aligned}
& A_{k}=\frac{1}{2}\left[r_{i, n}{ }^{2}-r_{1, n}{ }^{2}+\left\|B S_{1}\right\|^{2}-\left\|B S_{i}\right\|^{2}\right] \\
& B_{k}=\frac{1}{2}\left[r_{(i+1), n}{ }^{2}-r_{1, n}{ }^{2}+\left\|B S_{1}\right\|^{2}-\left\|B S_{(i+1)}\right\|^{2}\right] \\
& C_{k}=\left(x_{1}-x_{(i+1)}\right)\left(y_{1}-y_{i}\right)-\left(x_{1}-x_{i}\right)\left(y_{1}-y_{(i+1)}\right)
\end{aligned}
$$

for $k=1,2, \ldots N-1$
However, only 4 intersection points were selected as feasible intersection points to estimate the potential of MS positions which are marked as $a, b, l$ and $u$ points. These points will give the DOA measured relative to a known reference direction. The feasible range between MIMO $B S_{i}$ and these points is denoted as $\delta_{i, p}$ for $p=1,2, \ldots, \varphi_{\text {est }}$, where $\varphi_{\text {est }}$ represents the number of feasible intersection points for the estimated MS position.

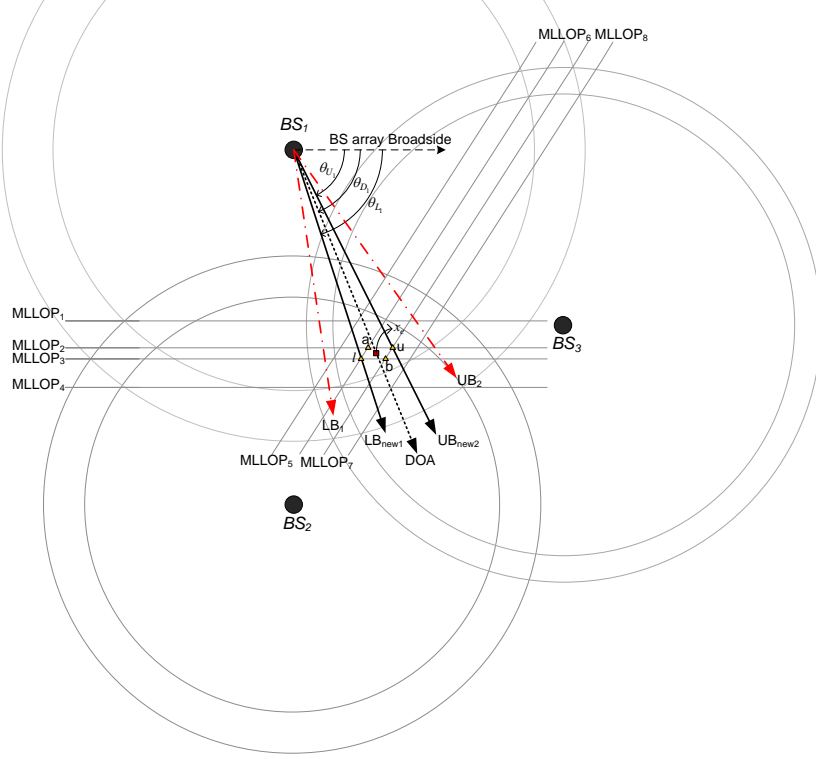

Figure 1 Geometry of HMLLOP-based location system

\subsection{Upper and Lower Bounds of DOA}

Figure 2 depicts an enlarged view of the geometry of an HMLLOPbased location which aims to find a new LB and UB for the measured DOA. As has been mentioned, beamforming is used to direct a signal in a particular direction. However, the DOA-based beamforming will be slightly biased due to NLOS errors. Dissimilar to range error, the error in DOA due to NLOS propagation can be either positive or negative. It can, therefore, be modeled as a Gaussian random variable with zero mean and variance. If the absolute maximum angular error on either side of the true line of position (DOA) is taken to be $\psi$, then the true DOA for $B S_{i}, \theta_{D_{i}}$ is always pointing within $\pm \psi$ of the measured DOAbased beamforming, $\theta_{D m_{i}}$. The LB and UB for DOA error are given as follows:

$$
\begin{array}{ll}
\theta_{L B_{i}}=\theta_{D_{i}}-\psi ; & \mathrm{LB} \\
\theta_{U B_{i}}=\theta_{D_{i}}+\psi ; & \mathrm{UB}
\end{array}
$$

Hence the measurement of DOA must lie between $\theta_{L B_{i}}$ and $\theta_{U B_{i}}$ and is given as follows:

$$
\theta_{L B_{i}} \leq \theta_{D m_{i}} \leq \theta_{U B_{i}}
$$

and: 
$\theta_{D m_{i}}=\arctan \left(\frac{y_{i}-y_{e}}{x_{i}-x_{e}}\right)$

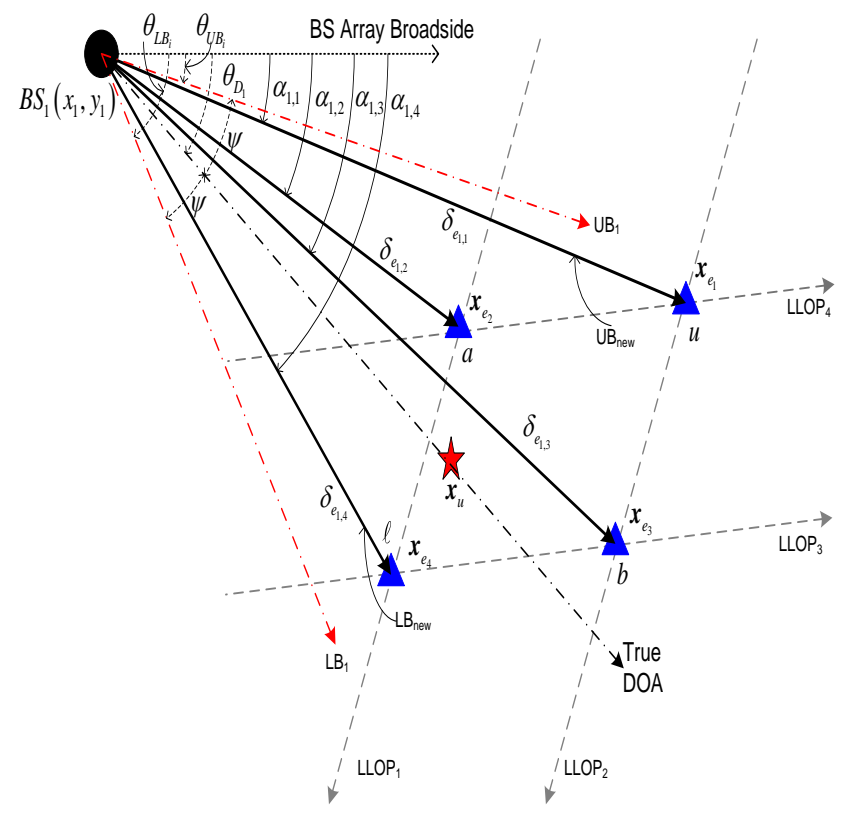

Figure 2 Geometry of HMLLOP-based location for determining lower and upper bounds of DOA

Note that the NLOS errors are included in $\theta_{D m_{i}}$. Therefore, this has introduced a non-linear equality constraint on MS location in the form of :

$\theta_{L B_{i}} \leq \arctan \left(\frac{y_{i}-y_{e}}{x_{i}-x_{e}}\right) \leq \theta_{U B_{i}}$

Next we calculate the range between $B S_{i}$ and each intersection point (feasible MS location):

$\delta_{i, p}=\sqrt{\left(x_{i}-x_{e_{p}}\right)^{T}\left(x_{i}-x_{e_{p}}\right)}$

where $\boldsymbol{x}_{i}=\left[x_{i}, y_{i}\right]^{T}$ represents the location of the $i$ th MIMO BS for $i=1,2, \ldots, N$ and $x_{e_{p}}=\left[x_{e_{p}}, y_{e_{p}}\right]^{T}$ denotes the location of the $p$ th feasible estimate of the MS .

In general, if $\alpha_{i, p}$ represents the orientation of the line joining the reference BS and the feasible intersection point of the $i$ th and $p$ th range linear lines, then considering the geometry of the range linear lines in Figure 2, the angle, $\alpha_{i, p}$, between $B S_{i}$ and the $p$ th feasible MS location can be calculated as [14]:

$\alpha_{i, p}=\tan ^{-1}\left(\frac{y_{e_{p}}-y_{i}}{x_{e_{p}}-x_{i}}\right)$

Then, we can calculate the new LB and UB based on the parameters obtained from the MLLOP algorithm. From (11), the LB and UB of the DOA can be determined as:
$\begin{array}{ll}\alpha_{L B_{i}}=\arg \min \left\{\alpha_{i, p}\right\} ; & L B \\ \alpha_{U B_{i}}=\arg \max \left\{\alpha_{i, p}\right\} ; & U B\end{array}$

for $p=1,2, \ldots, \varphi_{e s t}$.

Finally, the LB and UB of the DOA in (12) are compared with (6), and the new DOA can be determined as per the following conditions shown in Table 1.

Following a similar procedure for MLLOP TOA-based location in (5) and including the new DOA-based beamforming acquired from Table 1, the HMLLOP schemes can be expressed in matrix form as:

$$
\boldsymbol{H}_{H M L O P} \boldsymbol{x}=\boldsymbol{B}_{H M L O P}
$$

where:

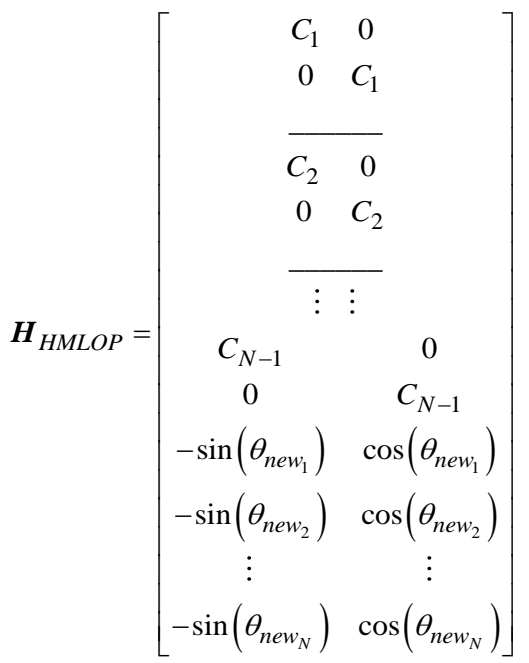

and:

$$
\boldsymbol{B}_{M L O P}=\left[\begin{array}{c}
B_{1}\left(y_{1}-y_{2}\right)-A_{1}\left(y_{1}-y_{3}\right) \\
A_{1}\left(x_{1}-x_{3}\right)-B_{1}\left(x_{1}-x_{2}\right) \\
\overline{B_{2}\left(y_{1}-y_{3}\right)-A_{2}\left(y_{1}-y_{4}\right)} \\
A_{2}\left(x_{1}-x_{4}\right)-B_{2}\left(x_{1}-x_{3}\right) \\
\vdots \\
B_{N-1}\left(y_{1}-y_{N-1}\right)-A_{N-1}\left(y_{1}-y_{N}\right) \\
A_{N-1}\left(x_{1}-x_{N)}\right)-B_{N-1}\left(x_{1}-x_{N-1}\right) \\
y_{1} \cos \left(\theta_{\text {new }_{1}}\right)-x_{1} \sin \left(\theta_{\text {new }_{1}}\right) \\
y_{2} \cos \left(\theta_{\text {new }_{2}}\right)-x_{2} \sin \left(\theta_{\text {new }_{2}}\right) \\
\vdots \\
y_{N} \cos \left(\theta_{\text {new }_{N}}\right)-x_{N} \sin \left(\theta_{\text {new }_{N}}\right)
\end{array}\right]
$$

The solution is then given by:

$$
\hat{\boldsymbol{x}}=\left(\boldsymbol{H}_{H M L O P}{ }^{T} \boldsymbol{H}_{H M L O P}\right)^{-1} \boldsymbol{H}_{H M L O P}{ }^{T} \boldsymbol{B}_{H M L O P}
$$


Table 1 Algorithm to determine new $\theta_{D_{i}}$

if $\theta_{D_{i}} \geq \alpha_{L B_{i}} \& \theta_{D_{i}} \leq \alpha_{U B_{i}}$
then $\theta_{D(\text { new })_{i}}=\theta_{D_{i}}$
elseif $\theta_{D_{i}<\alpha_{L B_{i}}}$
then $\theta_{D(\text { new })_{i}}=\alpha_{L B_{i}}$
elseif $\theta_{D_{i}}>\alpha_{U B_{i}}$
then $\theta_{D(\text { new })_{i}}=\alpha_{U B_{i}}$
end...

\subsection{PERFORMANCE AND SIMULATION RESULTS}

Simulations were conducted to determine the performance of the combined HMLLOP location technique by computer simulation and to compare location accuracy with existing positioning algorithms at various types of antenna mode configurations. In practice, the number of available BSs, typically $3-6 \mathrm{BSs}$, can be overheard by the MS at any time [15]. In this simulation, location estimation accuracy is checked for situations of up to $5 \mathrm{BSs}$ and simulations are performed under the assumption of a macro cellular environment. In this environment, the BS antenna is assumed to be situated at a higher level relative to the MS. Hence, the angular and range errors are caused by local scatters around the MS.

The simulated system parameters have been selected to be similar to the IEEE802.16e downlink system, and the dispersive delay properties of the channel introduce range errors of up to $600 \mathrm{~m}$ [16]. Therefore, the NLOS range errors are modeled as positive random variables having support over $[0,600 \mathrm{~m}]$ and generated according to a CDSM model [17]. We assume that for a BS equipped with an antenna array, multiple transmit beams send different pilots to each beam [18]. The receiving MS array can determine the scattered signal strength of each pilot and then recognize which transmit beam is employed. Therefore, the DOAs of multipath are resolvable according to the different pilot signals from the MS. The DOA error caused by the channel is considered to be a Gaussian distributed variable with a zero mean, and standard deviation (SD) is set to $3,5,10$ and 20 degrees [19]. The simulated location error has a total number of 1,000 different datasets and the estimation of MS position is obtained by averaging all 1,000 estimates. The TOA and DOA measurements are created by calculating the true distance from an MS position to a known BS with MIMO capability and each is corrupted by NLOS errors.

\subsection{Effects of DOA-Based Beamforming}

Firstly, a simulation is performed to investigate the effects of DOAbased beamforming on location estimation. In this simulation, only three BSs are considered. The range measurements are corrupted by NLOS errors with the CDSM model radius of scattering fixed at $100 \mathrm{~m}$ for all available BSs. Four curves are presented for the DOA $\mathrm{SD}$ at 3, 5, 10 and 20 degrees, as shown in Figure 3. As can be observed, with an increasing number of antenna mode configurations, the accuracy of the location estimation improves consistently, especially when large DOA errors are present at the MIMO BSs. On the other hand, the smallest DOA SD error performed almost linearly, in spite of the number of antenna mode configurations.
Meanwhile Figure 4 depicts the proposed DOA-based beamforming by utilizing the new LB and UB of the DOA. The simulation was performed to investigate the effectiveness of the proposed scheme at various degrees of DOA SD, i.e. 3, 5 and 10 degrees. The radius of the CDSM NLOS model is fixed at $100 \mathrm{~m}$. Basically, the proposed DOA scheme performed better than the current measurement of DOA. The improvement is likely to be greater at lower antenna mode configurations and almost identical when a larger antenna mode configuration is employed. In addition, we can observe that the proposed scheme works very well when the error in DOA SD is increased. In summary, the proposed DOA scheme supports the improvement of L\&P estimation, especially when there are large errors in DOA SD.

\subsection{Performance Analysis for the HMLLOP Algorithm}

We examine the performance of L\&P estimation among combined techniques. In this simulation we compare the HMLLOP algorithm with the HTD proposed by Sayed et al. [11], as described in Section 2.0. The NLOS parameter errors, such as radius of CDSM, $R_{d}$, and DOA SD, are fixed at $200 \mathrm{~m}$ and 5 degrees, respectively. The improvement in L\&P estimation provided by the combined schemes can be observed in the cumulative distribution function (CDF) of average RMSE error, as illustrated in Figure 5. The proposed HMLLOP algorithm generates more accurate location estimates than the HTD algorithm for the MIMO antenna mode configurations considered. For example, in the case of a MIMO2x1 antenna, the location error of the HMLLOP algorithm is less than $100 \mathrm{~m}$ for $78 \%$ of the time, whereas the HTD algorithm has the same location error for only $58 \%$ of the time. The same scenario can be observed for the other MIMO antenna mode configurations, where the performance of the HMLLOP scheme is always better than the HTD scheme.

\subsection{CONCLUSION}

This paper has proposed on the development of a combined L\&P system by combining range and angle measurements for a MIMO system. The proposed combined scheme, called an HMLLOP algorithm, is proposed as a method to determine the position of the MS based on a combination of an MLLOP range-based algorithm and DOA-based beamforming. The combined technique extends the basic idea of using an MLLOP scheme with additional information about DOA measurements when available. The proposed technique involves the use of multiple LOP instead of circular LOP and utilizes the bounds on DOA errors due to NLOS to find a solution for location estimation. Simulations of the HMLLOP algorithm were carried out to represent its performance in an outdoor environment, where TOA and DOA measurements are combined. The first simulation was done to investigate the effect of DOA-based beamforming on location estimation. The results show that with the extra parameter of DOA, the proposed technique supports improved accuracy of location estimation, especially for large errors in DOA standard deviation. The simulations also show that the combined HMLLOP algorithm provides better location accuracy than their range-based counterparts, in spite of any antenna mode configurations. In addition, compared to the existing combined HTD algorithm, the proposed technique achieved better performance when several MIMO antenna mode configurations were considered. More specifically, the results demonstrate that the average location error of the proposed algorithm is less than $85 \mathrm{~m}$ for $67 \%$ of the time, whereas the HTD algorithm is less than $115 \mathrm{~m}$ for the same error location, in the case of a MIMO $2 \times 1$ antenna mode configuration with NLOS parameter errors. 


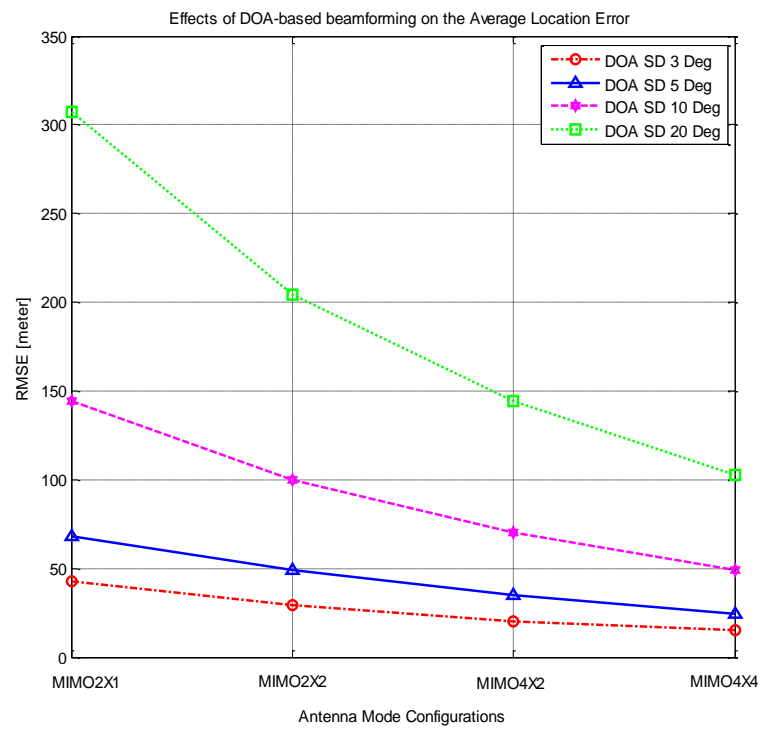

Figure 3 Effects of DOA standard deviation on location error with various numbers of mimo antenna mode configurations

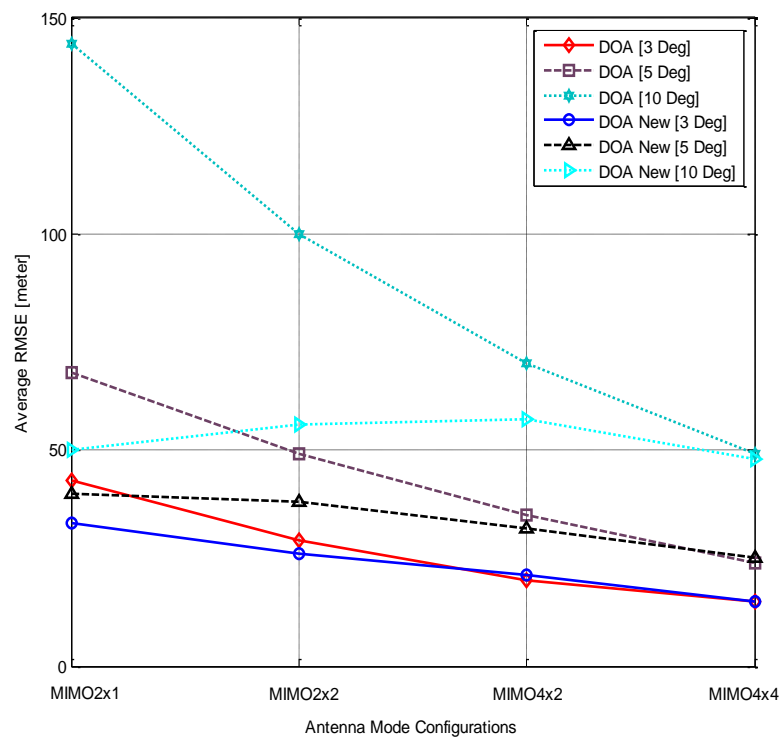

Figure 4 Comparison of average RMSE location error between proposed DOA and current DOA with several antenna mode configurations

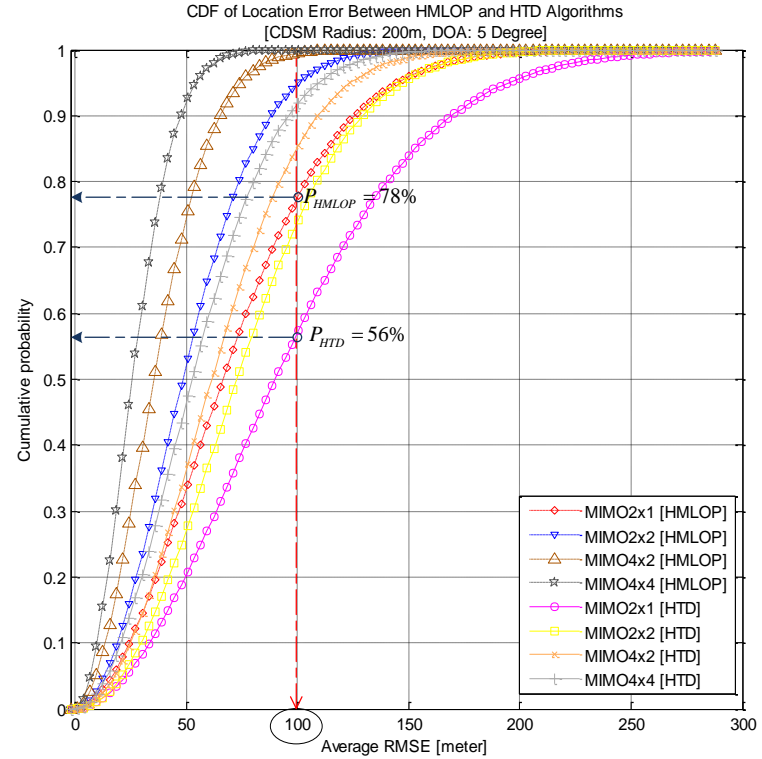

Figure $5 \mathrm{CDF}$ of location error of the HMLLOP and HTA algorithms for various antenna mode configurations (CDSM Radius: $200 \mathrm{~m}$, DOA SD: 5 Degrees)

\section{Acknowledgement}

Authors would like to thank the UTeM for funding this work in part under grants PJP/2012/FKEKK(9B)/S01105 and RAGS/2012/FKEKK/TK06/1 B00007.

\section{References}

[1] C. D. Gavrilovich, Jr. 2001. Broadband Communication on the Highways of Tomorrow. Communications Magazine, IEEE. 39: 146-154.

[2] A. Kupper. 2005. Location-based Services: Fundamentals and Operation: Wiley.

[3] C. Drane, M. Macnaughtan, and C. Scott. 1998. Positioning GSM Telephones. Communications Magazine, IEEE. 36: 46-54, 59.

[4] Jefry G.Andrews, Arunabha Ghosh, and R. Muhamed. 2007. Fundamental of WiMAX: Understanding Broadband Wireless Networking. Prentice Hall,

[5] White Paper. 2006. Understanding the Radio Technologies of Mobile WiMAX and Their Effect on Network Deployment Optimization.

[6] A. A. M. Isa. 2010. Enhancing Location Estimation Accuracy in WiMAX Networks. In MELECON 2010 - 2010 15th IEEE Mediterranean Electrotechnical Conference. 725-731.

[7] A. Awang Md Isa and G. Markarian. 2008. Positioning Technologies in Wireless Broadband Communications. In Cranfield Multi-Strand Conference (CMC2008), Cranfield United Kingdom.

[8] White Paper. 2008. Beamforming Boosts the Range and Capacity of WiMAX Networks.

[9] L. Ji, J. Conan, and S. Pierre. 2005. Joint Estimation of Channel Parameters for MIMO Communication Systems. In Wireless Communication Systems, 2005. 2nd International Symposium on. 22-26.

[10] A. Awang Md Isa and G. Markarian. 2011. MIMO Positioning for IMT Advanced Systems Based on Geometry Approach in NLOS Environments. Journal of Telecommunication, Electronic and Computer Engineering. 3 : 55-68,

[11] A. H. Sayed, A. Tarighat, and N. Khajehnouri. 2005. Network-based Wireless Location: Challenges Faced in Developing Techniques for Accurate Wireless Location Information. Signal Processing Magazine, IEEE. 22: 24-40.

[12] W. S. M. Jr, 2007. Determination of a Positioning Approximate Distance and Trilateration. Master's Thesis: Colorado School of Mines.

[13] N. William, William S. Murphy, Jr., and H. Willy. 1998. Statistical Methods in Surveying by Trilateration. vol. 27: Elsevier Science Publishers B. V. 209-227. 
[14] David Munoz , Frantz Bouchereau Lara Dr., Cesar Vargas, and R. Enriquez-Caldera. 2009. Position Location Techniques and Applications. Academic Press.

[15] L. Cong and W. Zhuang. 2004. Nonline-of-sight Error Mitigation in Mobile Location. In INFOCOM 2004. Twenty-third Annual Joint Conference of the IEEE Computer and Communications Societies. 659.

[16] C. L. F. Mayorga, F. della Rosa, S. A. Wardana, G. Simone, M. C. N. Raynal, J. Figueiras, and S. Frattasi, 2007. Cooperative Positioning Techniques for Mobile Localization in 4G Cellular Networks. In Pervasive Services, IEEE International Conference on. 39-44.
[17] Pieter Van Rooyen, Michiel Lötter, and D. V. Wyk. 2000. Space-time Processing for CDMA Mobile Communications. Kluwer Academic Publishers.

[18] M. S. M. Isa, R. J. Langley, S. Khamas, A. A. M. Isa, M. S. I. M. Zin, and F. M. Johar, 2012. Antenna Beam Steering using Sectorized Square EBG Journal of Telecommunication, Electronic and Computer Engineering, 4: 39-44.

[19] L. Nuaymi. 2007. WiMAX: Technology for Broadband Wireless Access. Wiley. 